\title{
DIOPHANTINE INEQUALITIES WITH MIXED POWERS $(\bmod 1)$
}

\author{
R. J. COOK
}

Abstract. A theorem of Heilbronn on the distribution of the sequence $n^{2} \theta(\bmod 1)$ is extended to sums of mixed powers.

1. Introduction. In 1948 Heilbronn [6] proved that for any $\varepsilon>0$ there exists $C(\varepsilon)$ such that for any real $\theta$ and $N \geqslant 1$ there is an integer $x$ satisfying

$$
1 \leqslant x \leqslant N \text { and }\left\|\theta x^{2}\right\|<C(\varepsilon) N^{-1 / 2+\varepsilon},
$$

where $\|\alpha\|$ denotes the difference between $\alpha$ and the nearest integer, taken positively. The result has been extended in several directions. In particular, in [2] an analogous result was obtained for the fractional parts of an additive form

$$
\theta_{1} x_{1}^{k}+\cdots+\theta_{s} x_{s}^{k}
$$

of degree $k$. Here we shall prove analogous results for mixed powers.

THEOREM 1 . Let $2 \leqslant k_{1} \leqslant \cdots \leqslant k_{s}$ be integers, $K_{i}=2^{k_{i}-1}$ and

$$
l=K_{1}^{-1}+\cdots+K_{s}^{-1} .
$$

If $l \geqslant 1$ then for any $\varepsilon>0, N>1$ and real $\theta_{1}, \ldots, \theta_{s}$ there exist integers $x_{1}, \ldots, x_{s}$ such that

$$
0<\max \left|x_{i}\right| \leqslant N \text { and }\left\|\theta_{1} x_{1}^{k_{1}}+\cdots+\theta_{s} x_{s}^{k_{s}}\right\|<C N^{-1+\varepsilon},
$$

where $C$ depends only on $k_{1}, \ldots, k_{s}$ and $\varepsilon$.

EXAmple 1. Let $k_{1}=2, k_{2}=k_{3}=3$. For any $\varepsilon>0$ there is a constant $C=C(\varepsilon)$ such that for any $N>1$ and real $\theta_{1}, \theta_{2}, \theta_{3}$ there are integers $x_{1}, x_{2}, x_{3}$ satisfying

$$
0<\max \left|x_{i}\right| \leqslant N \text { and }\left\|\theta_{1} x_{1}^{2}+\theta_{2} x_{2}^{3}+\theta_{3} x_{3}^{3}\right\|<C N^{-1+\varepsilon} .
$$

THEOREM 2 . Let $2 \leqslant k_{1} \leqslant \cdots \leqslant k_{s}$ be integers and let l be defined by (3). If $l<1$ then for any $\varepsilon>0, N>1$ and real $\theta_{1}, \ldots, \theta_{s}$ there exist integers $x_{1}, \ldots, x_{s}$ satisfying

Received by the editors May 27, 1975.

AMS (MOS) subject classifications (1970). Primary 10F40. 
(5) $0<\max \left|x_{i}\right| \leqslant N$ and $\left\|\theta_{1} x_{1}^{k_{1}}+\cdots+\theta_{s} x_{s}^{k_{s}}\right\|<C N^{-s /\left(s+(1-l) K_{s}\right)+\varepsilon}$, where $C$ depends only on $k_{1}, \ldots, k_{s}$ and $\varepsilon$.

EXAMPLE 2. Let $k_{1}=2, k_{2}=3$. For any $\varepsilon>0$ there is a constant $C=C(\varepsilon)$ such that for any $N>1$ and real $\theta_{1}, \theta_{2}$ there are integers $x_{1}, x_{2}$ satisfying

$$
0<\max \left|x_{i}\right| \leqslant N \text { and }\left\|\theta_{1} x_{1}^{2}+\theta_{2} x_{2}^{3}\right\|<C N^{-2 / 3+\varepsilon} .
$$

EXAMPLE 3. Let $s=k-1, k_{1}=2, k_{2}=3, \ldots, k_{s}=k$. For any $\varepsilon>0$ there is a constant $C=C(\varepsilon)$ such that for any $N>1$ and real $\theta_{1}, \ldots, \theta_{k-1}$ there are integers $x_{1}, \ldots, x_{k-1}$ satisfying

$$
0<\max \left|x_{i}\right| \leqslant N \text { and }\left\|\theta_{1} x_{1}^{2}+\theta_{2} x_{2}^{3}+\cdots+\theta_{k-1} x_{k-1}^{k}\right\|<C N^{-1+1 / k+\varepsilon} \text {. }
$$

Recently Chong and Liu [1] obtained an analogue of Heilbronn's theorem for the sum $P_{1}\left(x_{1}\right)+\cdots+P_{s}\left(x_{s}\right)$ of $s$ polynomials, having no constant term, where each of the polynomials is of exact degree $k$. They proved that if $s \geqslant K$ then the inequalities

$$
0<\max \left|x_{i}\right| \leqslant N, \quad\left\|P_{1}\left(x_{1}\right)+\cdots+P_{s}\left(x_{s}\right)\right\| \ll N^{-1 / K+\varepsilon}
$$

have a solution. However, if one of the polynomials, $P_{1}$ say, is of degree at most $k-1$ then Davenport [3] has shown that there is an integer $x$ satisfying

$$
1 \leqslant x \leqslant N \text { and }\left\|P_{1}(x)\right\| \ll N^{-1 /(K+1)+\varepsilon} .
$$

It therefore appears that an improved estimate for the sum of polynomials with differing degrees would require an improvement in the estimate when the degrees are all equal.

2. Preliminary lemmas and notation. We may suppose that $\varepsilon$ is a small positive number; let $\eta$ be a small positive number which can be chosen as an explicit function of $\varepsilon$. We may suppose that $N>N_{0}\left(k_{1}, \ldots, k_{s}, \varepsilon\right)$. By $F \ll G$ we mean that $|F|<C G$ where $C$ depends at most on $k_{1}, \ldots, k_{s}$ and $\varepsilon$. We write $e(z)$ for $\exp (2 \pi i z)$.

LEMma 1 (VINOGRADOv). Let $\Delta$ satisfy $0<\Delta<\frac{1}{2}$ and let a be a positive integer. There exists a function $\psi(z)$, periodic with period 1 , which satisfies

$$
\psi(z)=0 \text { for }\|z\| \geqslant \Delta
$$

and

$$
\psi(z)=\sum_{\nu=-\infty}^{\infty} a_{v} e(v z)
$$

where the $a_{v}$ are real numbers, $a_{0}=\Delta, a_{-v}=a_{v}$ and

$$
\left|a_{v}\right|<A \min \left(\Delta, v^{-a-1} \Delta^{-a}\right) \quad(v \neq 0),
$$

where $A$ depends only on $a$. 
This is a particular case of Lemma 12 of Chapter 1 of Vinogradov [7].

LEMMA 2 (DiRICHLET). Let $\theta$ be a real number and $Q \geqslant 1$. Then there exists an integer $q$ satisfying

$$
1 \leqslant q \leqslant Q \text { and }\|q \theta\| \leqslant Q^{-1} .
$$

See, for example, Theorem 36 of Hardy and Wright [5].

Lemma 3 (WeyL). Let $k \geqslant 2$ be an integer, $K=2^{k-1}$ and

$$
P(x)=\theta x^{k}+\theta_{k-1} x^{k-1}+\cdots+\theta_{1} x+\theta_{0}
$$

be a polynomial of exact degree $k$, i.e. $\theta \neq 0$. Let $B, N$ be real numbers with $N \geqslant 1$. Then for any $\eta>0$,

(12) $\left|\sum_{B \leqslant x \leqslant B+N} e(P(x))\right|^{K} \ll N^{\eta}\left(N^{K-1}+N^{K-k} \sum_{m=1}^{L} \min \left(N,\|m \theta\|^{-1}\right)\right)$

where $L=k ! N^{k-1}$.

See, for example, Lemma 1 of Davenport [4].

3. Proof of Theorem 1. Suppose that $K_{1}^{-1}+\cdots+K_{j}^{-1}<1 \leqslant K_{1}^{-1}+\cdots$ $+K_{j}^{-1}+K_{j+1}^{-1}$. Since $K_{1}^{-1}+\cdots+K_{j}^{-1}$ is a rational number whose denominator divides $K_{j}$, and $K_{j+1}$ is a multiple of $K_{j}$, it follows that $K_{1}^{-1}+\cdots+K_{j}^{-1}$ $+K_{j+1}^{-1}=1$. Therefore it is sufficient to prove Theorem 1 when $l=1$, since the general result follows on taking $x_{j+2}=x_{j+3}=\cdots=x_{s}=0$.

Let $M=N^{1-\varepsilon}$ and suppose that $\left\|\theta_{1} x_{1}^{k_{1}}+\cdots+\theta_{s} x_{s}^{k_{s}}\right\| \geqslant M^{-1}$ for $1 \leqslant x_{i}$ $\leqslant N, i=1, \ldots, s$. We apply Lemma 1 with $\Delta=M^{-1}$ and obtain

$$
\sum_{x_{1}=1}^{N} \cdots \sum_{x_{s}=1}^{N} \psi\left(\theta_{1} x_{1}^{k_{1}}+\cdots+\theta_{s} x_{s}^{k_{s}}\right)=0 .
$$

Therefore

$$
M^{-1} N^{s}+\sum_{v \neq 0} a_{v} \prod_{i=1}^{s} S_{v}(i)=0 \quad \text { where } S_{v}(i)=\sum_{x=1}^{N} e\left(v \theta_{i} x^{k_{i}}\right)
$$

Then

$$
M^{-1} N^{s} \ll \sum_{\nu=1}^{\infty}\left|a_{\nu}\right| \prod_{i=1}^{s}\left|S_{\nu}(i)\right| .
$$

Let $M_{1}=M^{1+\eta}$; then the contribution of those $v>M_{1}$ is $\ll N^{s-a \eta}$, and taking $a=\left[3 \eta^{-1}\right]$ we obtain

$$
M^{-1} N^{s} \ll \sum_{\nu=1}^{M_{1}}\left|a_{v}\right| \prod_{i=1}^{s}\left|S_{v}(i)\right| \ll M^{-1} \sum_{\nu=1}^{M_{1}} \prod_{i=1}^{s}\left|S_{v}(i)\right| .
$$

Since $l=1$ an application of Hölder's inequality gives

$$
N^{s} \ll \prod_{i=1}^{s}\left\{\sum_{\nu=1}^{M_{1}}\left|S_{\nu}(i)\right|^{K_{i}}\right\}^{1 / K_{i}}
$$


Therefore for some value $i$, for which we write $S_{v}(i), \theta_{i}, k_{i}, K_{i}$ as $S_{v}, \theta$, $k$ and $K$ respectively, we have $N^{K} \ll \sum_{v=1}^{M_{1}}\left|S_{v}\right|^{K}$. Applying Lemma 3 we have

$$
N^{K} \ll \sum_{\nu=1}^{M_{1}} N^{\eta}\left(N^{K-1}+N^{K-k} \sum_{m=1}^{L} \min \left(N,\|v m \theta\|^{-1}\right)\right) .
$$

The contribution of the first term on the right-hand side is $M_{1} N^{\eta} N^{K-1}$ $\ll N^{K-\varepsilon+2 \eta}$ which is $o\left(N^{K}\right)$ provided that $\eta<\varepsilon / 2$. Therefore

$$
N^{K} \ll N^{K-k+\eta} \sum_{\nu=1}^{M_{1}} \sum_{m=1}^{L} \min \left(N,\|v m \theta\|^{-1}\right) \ll N^{K-k+2 \eta} \sum_{h=1}^{H} \min \left(N,\|h \theta\|^{-1}\right)
$$

where $h=m v, H=M_{1} L$ and we have used the fact that the number of representations of $h$ in the form $m v$ is $\ll N^{\eta}$. Thus

$$
N^{k-2 \eta} \ll \sum_{h=1}^{H} \min \left(N,\|h \theta\|^{-1}\right) .
$$

We take $Q=N^{k-3 \eta}$ and choose an integer $q$ satisfying (10). Rearranging the sum on the right-hand side of (16) into blocks of length $q$ and estimating in the usual way, see, for example, [4, p. 13], we obtain

$$
N^{k-2 \eta} \ll\left(q^{-1} H+1\right)(N+q \log q) .
$$

Clearly $N=o\left(N^{k-2 \eta}\right)$,

$q \log q \ll Q N^{\eta / 2}=o\left(N^{k-2 \eta}\right)$ and $H \log q \ll M_{1} N^{k-1+\eta} \ll N^{k-\varepsilon+2 \eta}$ which is $o\left(N^{k-2 \eta}\right)$ provided that $\eta<\varepsilon / 4$.

Therefore

$$
N^{k-2 \eta} \ll q^{-1} H N
$$

so that

$$
q \ll M_{1} N^{2 \eta} .
$$

Then, for $N>N_{0}\left(k_{1}, \ldots, k_{s}, \varepsilon\right)$ we have $1 \leqslant q \leqslant N$ and

$$
\left\|q^{k} \theta\right\| \leqslant q^{k-1}\|q \theta\| \leqslant q^{k-1} Q^{-1} \ll M_{1}^{k-1} N^{-k+(2 k+1) \eta}<N^{-1}
$$

provided that $\eta$ is sufficiently small. Thus $x_{i}=q, x_{j}=0$ when $j \neq i$ gives a solution of the inequalities (4), which completes the proof of Theorem 1.

4. Proof of Theorem 2. If the inequalities (5) have no solution we take $t=s /\left(s+K_{s}(1-l)\right), M=N^{t-\varepsilon}$ and proceed as for Theorem 1. In place of (14) we obtain

$$
N^{s} \ll M_{1}^{1-l} \prod_{i=1}^{s}\left\{\sum_{v=1}^{M_{1}}\left|S_{v}(i)\right|^{K_{i}}\right\}^{1 / K_{i}}
$$

Thus for some $i$, for which we write $\theta_{i}$ as $\theta$, etc.,

$$
M_{1}^{l-1} N^{s} \ll\left\{\sum_{\nu=1}^{M_{1}}\left|S_{v}\right|^{K}\right\}^{s / K} .
$$


Applying Weyl's estimate we have

$$
\begin{aligned}
M_{1}^{K(l-1) / s} N^{K} & \ll \sum_{\nu=1}^{M_{1}}\left|S_{\nu}\right|^{K} \\
& \ll \sum_{\nu=1}^{M_{1}} N^{\eta}\left(N^{K-1}+N^{K-k} \sum_{m=1}^{L} \min \left(N,\|v m \theta\|^{-1}\right)\right) .
\end{aligned}
$$

Suppose first that

$$
M_{1}^{K(l-1) / s} N^{K} \ll M_{1} N^{\eta} N^{K-1},
$$

then

$$
N^{1-\eta} \ll M_{1}^{1+K(1-l) / s} \ll M_{1}^{1+K_{s}(1-l) / s}=M_{1}^{1 / t} .
$$

Therefore $1-\varepsilon / t \geqslant(1-\eta) /(1+\eta)$ and choosing $\eta$ sufficiently small we obtain a contradiction. Therefore

$$
M_{1}^{k(l-1) / s} N^{K} \ll N^{K-k+2 \eta} \sum_{h=1}^{H} \min \left(N,\|h \theta\|^{-1}\right)
$$

where $h=m v$ and $H=M_{1} L$. Hence

$$
M_{1}^{K(l-1) / s} N^{k-2 \eta} \ll \sum_{h=1}^{H} \min \left(N,\|h \theta\|^{-1}\right) .
$$

We take $Q=M_{1}^{K(l-1) / s} N^{k-3 \eta}$. Since

$$
k-K(1-l) t / s=k-K(1-l) /\left(s+K_{s}(1-l)\right) \geqslant k-1
$$

we have $Q \geqslant 1$ and so there exists an integer $q$ satisfying (10). Rearranging the sum on the right-hand side of (25) into blocks of $q$ terms we obtain

$$
M_{1}^{K(l-1) / s} N^{k-2 \eta} \ll\left(q^{-1} H+1\right)(N+q \log q) .
$$

Using (26) we have $N=o\left(M_{1}^{K(l-1) / s} N^{k-2 \eta}\right)$ and

$$
q \log q \ll Q N^{\eta / 2}=o\left(M_{1}^{K(l-1) / s} N^{k-2 \eta}\right) .
$$

Suppose that

$$
M_{1}{ }^{K(l-1) / s} N^{k-2 \eta} \ll H \log q \ll M_{1} N^{k-1+\eta}
$$

then

$$
N^{1-3 \eta} \ll M_{1}^{1+K(1-l) / s} \ll M_{1}^{1 / t} .
$$

Therefore $1-3 \eta \leqslant(1+\eta)(1-\varepsilon / t)$ which gives a contradiction, provided that $\eta$ is sufficiently small. Therefore $(28)$ is false and so

$$
M_{1}^{K(l-1) / s} N^{k-2 \eta} \ll q^{-1} H N
$$

which implies 


$$
q \ll N^{2 \eta} M_{1}^{1+K(1-l) / s} \ll N^{2 \eta} M_{1}^{1 / t} .
$$

Then $1 \leqslant q \leqslant N$ and

$$
\begin{aligned}
\left\|q^{k} \theta\right\| & \leqslant q^{k-1}\|q \theta\| \leqslant q^{k-1} Q^{-1} \\
& \ll N^{-k+(2 k+1) \eta} M_{1}^{(k-1) / t+K(1-l) / s} \ll N^{-t+\varepsilon}
\end{aligned}
$$

since

$$
-k+t\left\{\frac{k-1}{t}+\frac{K(1-l)}{s}\right\} \leqslant-1+\frac{t K_{s}(1-l)}{s}=-t,
$$

and this completes the proof of Theorem 2 .

\section{REFERENCES}

1. K.-K. Chong and M. C. Liu, The fractional parts of a sum of polynomials, Monatsh. Math. (to appear).

2. R. J. Cook, The fractional parts of an additive form, Proc. Cambridge Philos. Soc. 72 (1972), 209-212. MR 45 \#6764.

3. H. Davenport, On a theorem of Heilbronn, Quart. J. Math. Oxford Ser. (2) 18 (1967), 339-344. MR 36 \#6355.

4. - Analytic methods for Diophantine equations and Diophantine inequalities, Ann Arbor, Ann Arbor, Mich., 1963. MR 28 \#3002.

5. G. H. Hardy and E. M. Wright, An introduction to the theory of numbers, 4 th ed., Oxford Univ. Press, Oxford, 1965.

6. H. Heilbronn, On the distribution of the sequence $n^{2} \theta(\bmod 1)$, Quart. J. Math. Oxford Ser. 19 (1948), 249-256. MR 10, 284.

7. I. M. Vinogradov, The method of trigonometrical sums in the theory of numbers, Trudy Mat. Inst. Steklov. 23 (1947); English transl., Interscience, New York and London, 1954. MR 10, 599; 15, 941 .

Department of Pure Mathematics, University of Sheffield, Sheffield S10 2TN, EngLAND 